Andrews University

Digital Commons @ Andrews University

Honors Theses

Undergraduate Research

4-5-2019

\title{
Isolation and Characterization of Novel Arginine-Derived Heterocyclic Amines
}

Nathaniel Srikureja

Andrews University, srikureja@andrews.edu

Follow this and additional works at: https://digitalcommons.andrews.edu/honors

Part of the Biochemistry, Biophysics, and Structural Biology Commons, and the Chemistry Commons

\section{Recommended Citation}

Srikureja, Nathaniel, "Isolation and Characterization of Novel Arginine-Derived Heterocyclic Amines" (2019). Honors Theses. 213.

https://dx.doi.org/10.32597/honors/213

https://digitalcommons.andrews.edu/honors/213

This Honors Thesis is brought to you for free and open access by the Undergraduate Research at Digital Commons @ Andrews University. It has been accepted for inclusion in Honors Theses by an authorized administrator of Digital Commons @ Andrews University. For more information, please contact repository@andrews.edu. 


\author{
J.N. Andrews Scholars \\ Andrews University Honors Program \\ Honors Thesis
}

Isolation and Characterization of Novel Arginine-Derived Heterocyclic Amines

\author{
Nathaniel Srikureja
}

April 5, 2019

Advisor: Dr. Ryan T. Hayes, PhD

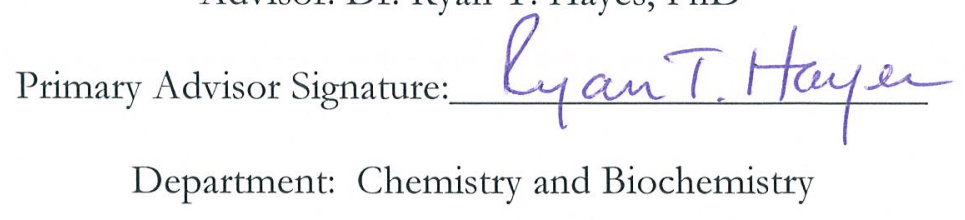




\begin{abstract}
Most known heterocyclic amines (HCAs) are potent mutagens present at significant concentrations in cooked meats, and can be synthesized from burned mixtures of L-creatin(in)e with various amino acids. We hypothesize that novel mutagenic HCAs may be synthesized from a heated plant-based food simulating binary amino acid mixture: L-arginine and L-threonine. A two-part solid phase extraction was used to separate nitrogen heterocycles from byproducts followed by prepHPLC and the Ames mutagenicity assay using Salmonella TA98 to determine the mutagenicity of various isolates. The results indicate that this binary amino acid mixture does not yield a mutagenic sample.
\end{abstract}




\section{Introduction}

Current epidemiological studies indicate that 30-35\% of cancer-related deaths can be directly linked to diet; the other $65-70 \%$ linked to tobacco, infection, and several other minor sources. In fact, only 5-10\% of novel cancer cases are due to genetic inheritance (Anand et al, 2008). Lifestyle factors, especially diet, have important roles in the initiation of biological malignancy. Therefore, the characterization of carcinogens, assessment of their risk to humans, and development of techniques to reduce exposure to these compounds remain an important modern research goal (Puangsombat, 2010; Sugimura et al, 2004; Zheng \& Lee, 2009).

The majority of dietary carcinogens are either naturally occurring compounds and toxins or compounds created as a byproduct of food processing methods. This latter group includes both polycyclic aromatic hydrocarbons (PAHs) and heterocyclic amines (HCAs). HCAs or aminoimidoazaarines, are planar nitrogen containing compounds with at least one heterocyclic ring generally an imidazole or azaarene ring - and an exocyclic amine group, formed ubiquitously across protein-containing foods, and especially in cooked and charred meats. Research on HCAs in the last 40 years has revealed hundreds of mutagenic and several carcinogenic compounds formed specifically in meat-based foods during cooking, thus increasing the strong correlation between the consumption of highly processed meats and the development of cancer (Alaejos \& Afonso, 2011; Felton et al, 1986). HCAs represent a significant health concern. Many HCAs are formed through the reaction between creatin(in)e - an amino acid derivative found only in meats - and various amino acids at temperatures at $150-300{ }^{\circ} \mathrm{C}$ (Figure 1). Upon pon ingestion, HCAs exert their potent oncogenic effect by binding to DNA, forming both adducts to guanine and intercalating disks, thus hampering proper DNA replication and transcription through well-known tumorigenesis pathways (Sugimura et al, 2004; Shut \& Snyderwine, 1999).

Cooked plant-based foods also present a potential source of HCAs. However, epidemiological studies suggest that the ingestion of cooked or charred plant-based foods carries only a fraction of the risk of tumorigenesis that similarly prepared meat-based foods carry. Notwithstanding, recent studies report finding carcinogenic compounds in plant-based foods

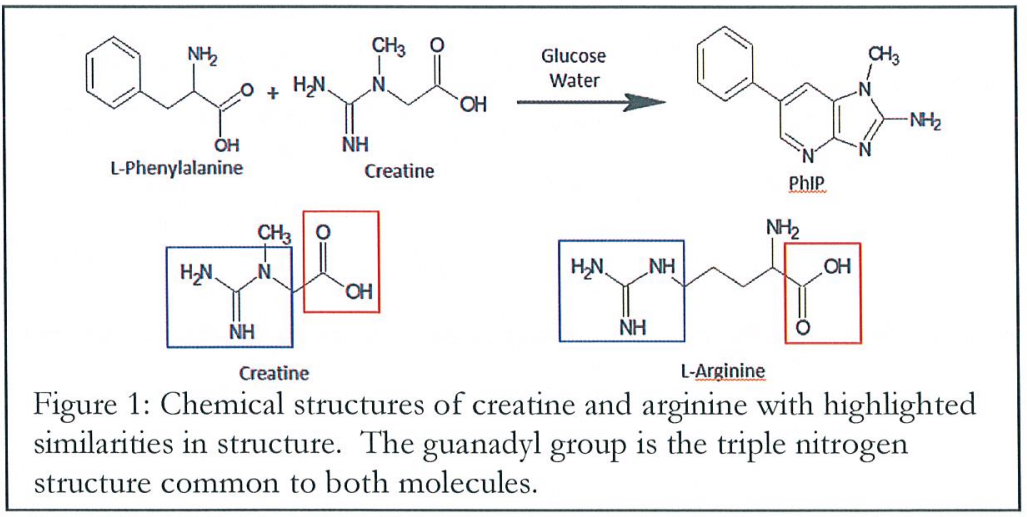
or plant-simulating samples, similar to known meat-based heterocyclic amines have been observed. For instance, Knize et al observe that burned binary amino acid mixtures produce mutagenic samples, and attribute this activity to a set of novel uncharacterized HCAs (1994).

The formation of potentially mutagenic HCAs within cooked plant-based foods, in the absence of creat(in)ine, is not farfetched. Knize et al observed the formation of mutagenic compounds within heated grain products (1994). In heated conditions, L-arginine is the most likely substitute for possible, on the basis that arginine and other amino acids. Creatin(in)e and L-arginine share several significant molecular similarities: both molecules possess a guanidyl group and a carboxylic acid group, potentially allowing L-arginine to react with other amino acids in a manner 
analogous to creat(in)ine (Figure 1). Indeed, the binary amino acid mixture with significant levels of mutagenicity contain L-arginine and some other amino acids. Within this list, the binary amino acid mixture containing L-arginine and L-threonine has the greatest mutagenicity. Previous research projects under Dr. Hayes by Tyler Pender, J.C. Lynch, Zach Reichert, Andrew Stewart, and Rayford Alva, Irene Hwang, and Tori Kim have focused on developing burning methods, extraction procedures, MS analysis, HPLC optimization, and Ames Test validation to help determine the identity of heterocyclic amines present in burned amino acid mixtures containing L-arginine and Lphenylalanine in the presence of glucose, heated to various temperatures (Pender, 2013; Reichert, 2017). This research project has similar goals, but intends to analyze a novel binary amino acid model system, containing L-arginine and L-threonine without a carbohydrate additive, and analyzing the results of the burned sample to isolate and characterize mutagenic samples.

\section{Methodology}

The methodology was adapted from Knize et al, which is crudely based on the widely accepted Gross and Grueter extraction method to isolate heterocyclic amines from a mixture of amino acids. (Felton et al 1986; Gibis, 2009; Knize et al 1994). Evidence suggests that the potential mutagens within burned plant-based amino acid samples share several important similarities with previously characterized heterocyclic amines from meat-based samples: compounds should possess one or more nitrogen-containing heterocycles, a guanidyl group, and one or more free exocyclic amino groups. These chemical moieties allow crude estimations of chemical properties that predict successful separation techniques.

Burning

A significant problem with similar previous research was prohibitively small concentrations of heterocyclic amines resulting from the burned binary amino acid mixture, limiting the ability to adequately analyze compounds produced by the burnings (Reichert, 2016). A significant portion of the yield was characteristically lost to the separation and extraction process, as well as to the Ames mutagenicity test. In other words, current yield estimates indicate that smaller-scale burnings of the L-arginine and L-threonine mixture do not yield enough product for characterization. To fulfill the goals of this research, larger samples were prepared for HPLC separation by increasing the amounts of reagents used. L-arginine and L-thronine were purchased from Sigma Aldrich chemicals, and stored at room temperature in the laboratory. Approximately $0.1 \mathrm{~mol} \mathrm{~L}$-threonine $(17.4 \mathrm{~g})$ and 0.1 mol L-threonine $(11.9 \mathrm{~g})$ were combined in a round bottom flask and heated at $210^{\circ} \mathrm{C}$ for 1 hour with occasional stirring.

\section{Extraction}

The burned mixture was dissolved in $1 \mathrm{M} \mathrm{NaOH}$, and mixed thoroughly with a diatomaceous earth slurry, which allowed the polar molecules to adhere to the beads of diatomaceous earth. Nonpolar compounds were extracted from the slurry by triplicate rinse using $5 \% \mathrm{v} / \mathrm{v}$ toluene in dichloromethane. The resulting extract was placed on an SPE-PRS (propylsulfonic) acid-silica gel solid-phase Bond-Elut extraction column, designed specifically for the isolation of nitrogen containing compounds. The column was eluted using $10 \mathrm{~mL}$ of $10 \% \mathrm{v} / \mathrm{v}$ ammonium hydroxide in methanol solution. The first $2 \mathrm{~mL}$ of eluent were collected, filtered through a $15 \mathrm{~mm}, 0.45 \mu \mathrm{m}$ pore size Phenomenex P'TFE membrane syringe filter, rotary evaporated, weighed and then dissolved in methanol for further analysis by HPLC. The extraction yield was 
calculated after rotary evaporation of the $2 \mathrm{~mL}$ of effluent was extracted from the PRS column. Approximately $10 \%$ of the extraction yield was set aside for PrepHPLC analysis.

\section{HPLC Analysis and Sample Fractioning}

PrepHPLC coupled with UV/Vis-spectroscopy was used to isolate and partially characterize components of the sample mixture. Preparatory HPLC is a sensitive and reliable technique used to separate compounds based on various molecular characteristics. A dissolved sample is injected into a liquid solvent stream passing through an HPLC column and a UV-vis spectrometer. The HPLC column is a packed column that separates compounds in a passing liquid phase based on their various chemical properties. An HPLC schematic is illustrated in Figure 2.

Our laboratory used the Waters PrepLC 4000 system with a 150 x $30 \mathrm{~mm}$ Phenomenex Luna 5 C18 column with a $5 \mu \mathrm{m}$ particle packing size, a $65 \% \mathrm{v} / \mathrm{v}$ acetonitrile in deionized water as mobile phase, $20 \mathrm{~mL} / \mathrm{min}$ flow rate, a 9-minute total run-time, an autosampler was used to automatically inject $500 \mu \mathrm{L}$ aliquots of the methanol-dissolved sample. Between injections, a 1minute wash procedure pumps $65 \% \mathrm{v} / \mathrm{v}$ aqueous acetonitrile through the C18 column at the same flow rate. The spectrogram was compared to negative controls: L-arginine, L-threonine, and a solvent control, as well as to a positive control: PhIP, a known heterocyclic amine, which can be synthesized and extracted from a burned mixture of creat(in)e and L-phenylalanine using a modified Gross-Greuter methodology. PhIP absorbs maximally around $315 \mathrm{~nm}$, so fractions of the sample were collected to separate peaks around this wavelength; fractions were collected at 0-3 minutes, 3-6 minutes, and 6-9 minutes. The fractions were rotary evaporated and then weighed before analysis using the Ames mutagenicity assay.

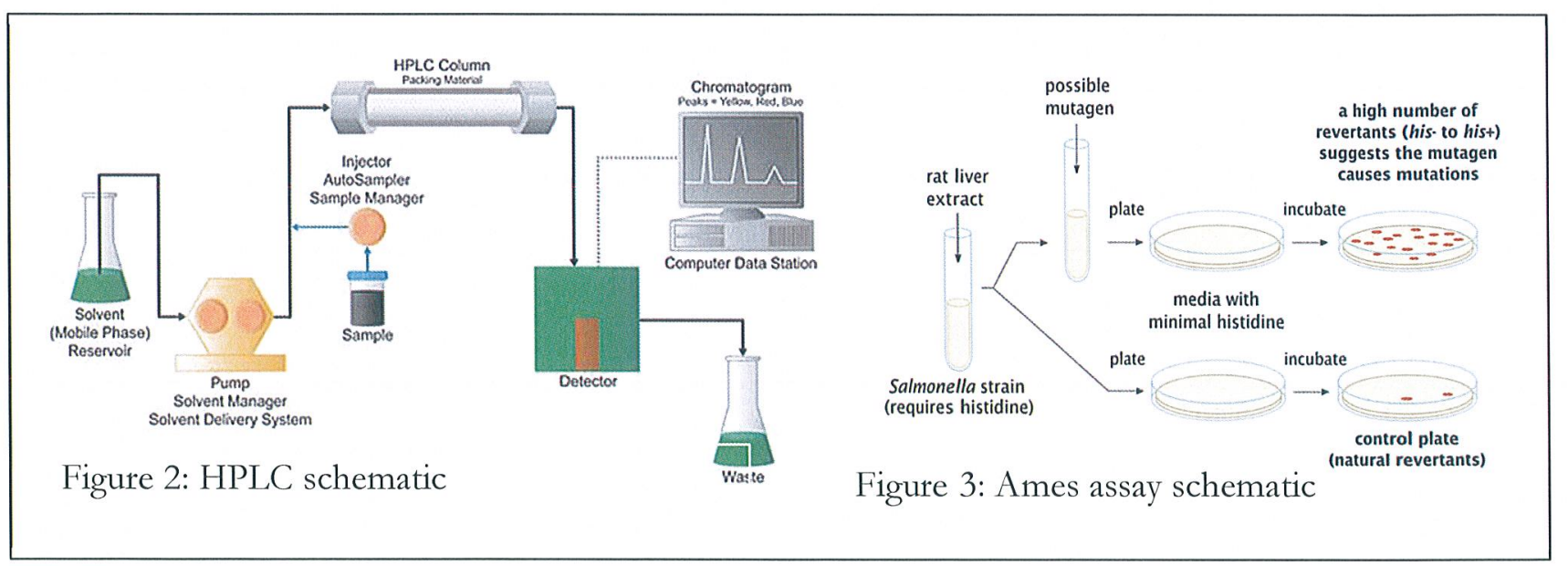

\section{Ames Mutagenicity Assay}

The mutagenicity of the burned samples and fractioned isolates were determined using the Ames test. Generally, a strain of Salmonella unable to synthesize histidine is grown on a histidinelacking medium with or without the suspected mutagen. Growth of bacteria on this medium is indicative of a genetic change which allows bacteria to synthesize histidine de novo; colony-forming units (CFUs) found after incubation were referred to as revertants (Figure 3). Subsequently, a significant increase in the rate of successful bacterial growth - rate of revertants - in the presence of 
the suspected mutagen compared to a control signifies mutagenic activity of the sample.

Furthermore, mutagenic activity should be dose-dependent in small amounts.

S-9 activated Salmonella TA98 was incubated for 48 hours in bis- medium with or without the extracted sample or isolated fraction dissolved in methanol at a known concentration. Counts of revertants per gram amounts of sample added per plate were measured by manually counting CFUs after the 48-hour period. The mutagenicity of a given sample was determined in triplicate. The Ames test was performed not only on an aliquot of the sample directly after the extraction method, but also on each of the fractions collected from the PrepHPLC.

PRS Column Regeneration

The Bond-Elut column used for the PRS extraction after burning is sold intended for single use, and thus does not come with instructions on cleaning and regeneration between extractions. Furthermore, when used without any regeneration or rinse method, significant differences in HPLC/UV-Vis spectroscopy in samples between consecutive extractions demonstrate a lack of consistency. Thus, the columns either must be thrown out after a single use - a relatively expensive practice - or a consistent washing method be implemented. A secondary goal of this research project was to design and implement a column regeneration method which would allow consistent reuse of the column.

$1.000 \mathrm{~mL}$ of a $10.0 \mathrm{mg} / \mathrm{mL}$ solution of PhIP in methanol was loaded onto the PRS column. The column was eluted using $10 \mathrm{~mL} \mathrm{10 \%} \mathrm{ammonium} \mathrm{hydroxide} \mathrm{in} \mathrm{methanol} \mathrm{solution.} \mathrm{The} \mathrm{first} 2$ $\mathrm{mL}$ of eluent were collected and rotary evaporated and then weighed after filtration using a syringe filter. Then, $5 \mathrm{~mL}$ of $1 \mathrm{M} \mathrm{HCl}$ was then used to rinse the column slowly to regenerate the cationexchange beads, and the extraction procedure was repeated on the regenerated column. HPLC was used to demonstrate the presence of PhIP in the eluent, and the mass of the extract was compared between extraction procedures to ensure that the regeneration process did not decrease the efficacy of the column between extractions.

Creatine and L-phenylalanine Burning

The reaction between creat(in)ine and phenylalanine at high temperatures is a wellcharacterized reaction and yields PhIP under a wide range of conditions (Manabe et al, 1992). Thus, the burning, extraction, and HPLC methodology with a reduced, $40 \mu \mathrm{L}$ injection size was performed burning a 1:1 molar ratio of $2 \mathrm{mmol}$ creatine and L-phenylalanine in otherwise identical conditions. This reaction was analyzed to determine the viability of our methodology, and provide a crude baseline for the burnings between L-arginine and L-threonine. No fractions were collected from the PrepHPLC, instead, $1.000 \mathrm{~mL}$ of each sample were spiked with a $500 \mu \mathrm{L}$ aliquot of a $100 \mu \mathrm{g} / \mathrm{mL}$ $\mathrm{PhIP}$ in methanol, and the PrepHPLC procedure was repeated. The UV-vis light absorption was measured around $315 \mathrm{~nm}$ and 4.0-minute retention time for both the unspiked and spiked samples, and the absorbance values for the unspiked samples were compared after correcting for sample dilution in the spiked samples.

\section{Results}

Creatine and L-phenylalanine Burning 
Four samples were prepared from the reaction between approximately $2 \mathrm{mmol}$ creatine and L-phenylalanine. For each sample, both the first $2 \mathrm{~mL}$ and last $8 \mathrm{~mL}$ of effluent from the PRS column was analyzed by PrepHPLC. "A" samples were derived from the first $2 \mathrm{~mL}$ of effluent off the PRS column, while "B" samples are derived from the last $8 \mathrm{~mL}$ of effluent off the PRS column. The absorbance at $315 \mathrm{~nm}$ and a retention time of approximately $4.0 \mathrm{~min}$ for both the spiked and unspiked samples are listed in Table 1. The absorbance of the unspiked samples were corrected by decreasing the value by a factor of 1.5 , equivalent to the dilution factor of the samples upon spiking. Assuming the linear relationship between absorbance and concentration holds for the concentrations of $\mathrm{PhIP}$ observed in these samples, the concentration of $\mathrm{PhIP}$ in the original samples - the $2 \mathrm{~mL}$ and $8 \mathrm{~mL}$ aliquots after extraction - was found by solving [1] $A=\epsilon c l ; \frac{A_{\text {spiked }}}{A_{\text {unspiked }}}=\frac{[P h I P]+100 \mu \mathrm{g} / \mathrm{mL}}{[P h I P]} ;[\mathrm{PhIP}]$ in $\mu \mathrm{g} / \mathrm{mL}$ and then correcting for the volume of the original sample in either " $A$ " or " $B$ " samples $-2 \mathrm{~mL}$ or 8 $\mathrm{mL}$. The mean concentration and standard deviation of PhIP in "A" samples derived from the first $2 \mathrm{~mL}$ of effluent from the PRS column was $26 \pm 13 \mu \mathrm{g} / \mathrm{m}$; the mean concentration and standard deviation of PhIP in "B" samples derived from the last $8 \mathrm{~mL}$ of effluent was $13 \pm 1 \mu \mathrm{g} / \mathrm{mL}$.

Table 1: Absorbance of spiked and unspiked creatine and L-phenylalanine burn samples, and calculated concentration of [PhIP].

\begin{tabular}{|c|c|c|c|c|}
\hline & \multicolumn{2}{|c|}{ Unspiked Absorbance /AL } & \multirow[b]{2}{*}{ Spiked Absorbance / AU } & \multirow[b]{2}{*}[\mathrm{PhIP}]{$/ \mu \mathrm{g} \mathrm{mL^{-1 }}$} \\
\hline & Initial & Corrected & & \\
\hline $4 \mathrm{~A}$ & 0.03 & 0.02 & 0.05 & 33 \\
\hline $5 \mathrm{~A}$ & 0.01 & 0.01 & 0.04 & 10 \\
\hline $7 \mathrm{~A}$ & 0.03 & 0.02 & 0.05 & 33 \\
\hline $6 \mathrm{~B}$ & 0.04 & 0.03 & 0.05 & 14 \\
\hline $4 \mathrm{~B}$ & 0.03 & 0.02 & 0.04 & 13 \\
\hline
\end{tabular}

\section{PRS Column Regeneration}

PrepHPLC analysis confirmed the presence of a peak within the extracts from the PRS column after the novel regeneration technique at $315 \mathrm{~nm}$ and a retention time of approximately 4.0 minutes. Both the 0-2 $\mathrm{mL}$ and 2-8 $\mathrm{mL}$ extracts possessed this peak within their UV-vis light absorption spectrum. This peak matches the characteristic peak UV-vis light absorption of PhIP. Since the data does not display a downward trend, indicating a loss of product throughout the consecutive extractions, the coefficient of variance (COV) of the mass of the dry extract was used as a normalized measurement of the collected deviation of a data set from its mean; in other words, the COV is an index of the variation within the data set, and is a good indicator of statistical consistency. The COV was determined separately for the set of extracts from the first $2 \mathrm{~mL}$ and the last $8 \mathrm{~mL}$ of effluent by dividing the standard deviation value by the mean value for each set respectively. The data collected, as well as the coefficient of variance is listed in Table 2 . The observed COV for the dried extract from the first $2 \mathrm{~mL}$ of effluent is 0.2689 , whereas the observed COV for the dried extract from the last $8 \mathrm{~mL}$ of effluent is 0.3519 . A significant portion of the inconsistency observed is due to the small mass amounts of PhIP used to test the column; for increasingly small values, equal variances dues to instrumental error introduce increasingly larger amounts of relative error. However, the observed COV values, combined with the HPLC analysis, indicate that PhIP is being extracted from the column appropriately, and that 
rinsing the column with $1 \mathrm{M} \mathrm{HCl}$ is an effective regeneration method which allows limited column reuse.

Table 2: Mass of dry extract from the PRS column from either the first $2 \mathrm{~mL}$ of effluent, or the last $8 \mathrm{~mL}$ of effluent. Seven total trials are performed. The mean values, standard deviations (StDev), and coefficients of variance (COV) are shown.

\begin{tabular}{|c|cc|}
\hline \multicolumn{1}{c}{} & \multicolumn{2}{c|}{ Mass Dry Extract $/ \mathrm{g}$} \\
\cline { 2 - 3 } Trial 1 & $0-2 \mathrm{~mL}$ & $2-8 \mathrm{~mL}$ \\
\cline { 2 - 3 } Trial 2 & 0.0092 & 0.0099 \\
Trial 3 & 0.0030 & 0.0086 \\
Trial 4 & 0.0098 & 0.0015 \\
Trial 5 & 0.0081 & 0.0076 \\
Trial 6 & 0.0092 & 0.0091 \\
Trial 7 & 0.0096 & 0.0088 \\
& 0.0096 & 0.0066 \\
mean & 0.0084 & 0.0074 \\
StDev & 0.0022 & 0.0026 \\
COV & 0.2689 & 0.3519 \\
\hline
\end{tabular}

\section{L-Arginine and L-Threonine Burning}

Three arginine-threonine burn samples were produced. The mass of reagents used, the extraction yield, the mass of the sample analyzed by HPLC, and the mass of each fraction produced are listed in Table 3. The extraction yield was approximately $0.3 \%$; the mass of each fraction was between $2.9 \mathrm{mg}$ and $18.9 \mathrm{mg}$, which accounted for only $0.01 \%$ to $0.06 \%$ of the original mass of the reagents. Much of the reactive material either remained unreacted and was correctly discarded, or perhaps did not react and was incorrectly removed by conservative or improper extraction methods.

Table 3: Reagent mass and yields for burnings, extraction, and fractions of L-arginine and L-threonine.

\begin{tabular}{|c|lllllll|}
\hline \multicolumn{2}{c}{} & \multicolumn{2}{c}{ Reagent Mass /g } & Extraction & Mass Analyzed by & \multicolumn{3}{c|}{ Fraction Mass /g } \\
Burn 1 & 17.410 & 11.912 & 0.1551 & 0.01449 & 0.0105 & 0.0189 & 0.0107 \\
Burn 2 & 17.437 & 11.920 & 0.1098 & 0.01112 & 0.0029 & 0.0056 & 0.0165 \\
Burn 3 & 17.426 & 11.937 & 0.1002 & 0.01120 & 0.0041 & 0.0062 & 0.0033 \\
\hline
\end{tabular}

PrepHPLC analysis was performed on several negative controls. The methanol solvent control had no significant peaks above $200 \mathrm{~nm}$. The L-arginine control, Figure 4, had a maximum absorption at $209 \mathrm{~nm}$ and a retention time of 2.7 minutes; the L-threonine control, Figure 5, had a maximum absorption at $236 \mathrm{~nm}$ and a retention time of 2.6 minutes. 


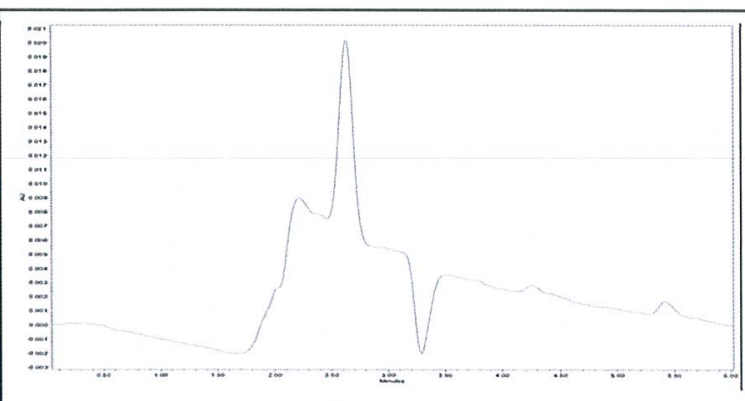

Figure 4: Negative control; Chromatogram of Larginine at $209 \mathrm{~nm}$; maximum absorbance at 2.7 minutes

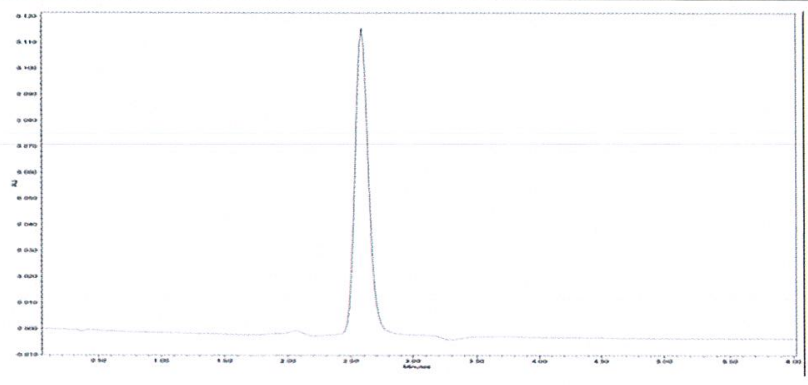

Figure 5: Negative control; Chromatogram of L-threonine at $236 \mathrm{~nm}$; maximum absorbance at 2.6 minutes
PrepHPLC analysis was also performed on a positive control. A 10 $\mu \mathrm{g} / \mathrm{mL}$ solution of PhIP was analyzed, and had a maximal absorption at $315 \mathrm{~nm}$ and a retention time of 3.8 minutes (Figure 6). Even though PhIP may not be found in the samples of burned L-arginine and Lthreonine, any novel HCA should have a similar molecular profile, and should have a UV-vis light absorption profile and retention time similar to a known HCA.

PrepHPLC analysis of each of the burn samples demonstrated the presence of large amounts of unreacted amino acids in the burn samples, as well as an unknown compound with maximum absorbance around $283 \mathrm{~nm}$ and a retention time of 3.6 minutes (Figure 7). By taking fractions at 0-3 minutes and 3-6 minutes, the sample was roughly separated into unreacted amino acids - appearing in the fraction from 0-3 minutes, and the unknown compound - appearing in the fraction from 3-6 minutes. Each fraction was then analyzed for mutagenicity using the Ames mutagenicity assay.

The Ames mutagenicity assay was performed on all 9 fractions and on the 3 burn samples before HPLC analysis. Each assay was performed either in duplicate or triplicate at low and high dosages. The number of revertants observed for each sample at each dosage was measured, and compared to a negative solvent control and a PhIP positive control. Figure 8 displays the results of the Ames assay. None of the fractions collected displayed any significant increase in mutagenicity above the negative control. To reject the null hypothesis that the fractions collected alter the mutagenicity from the solvent negative control, a two-tailed, two sample equal variance t-test was performed, comparing the observed revertant rate of each sample set to the revertant rate of the negative solvent control. p-values range from 0.05 to 0.85 ; no sample set yielded a p-value less than 0.05; no sample demonstrated a significant increase in mutagenicity above baseline. Therefore, at the concentrations tested, the unknown compound does not have mutagenic activity towards TA98 Salmonella. 

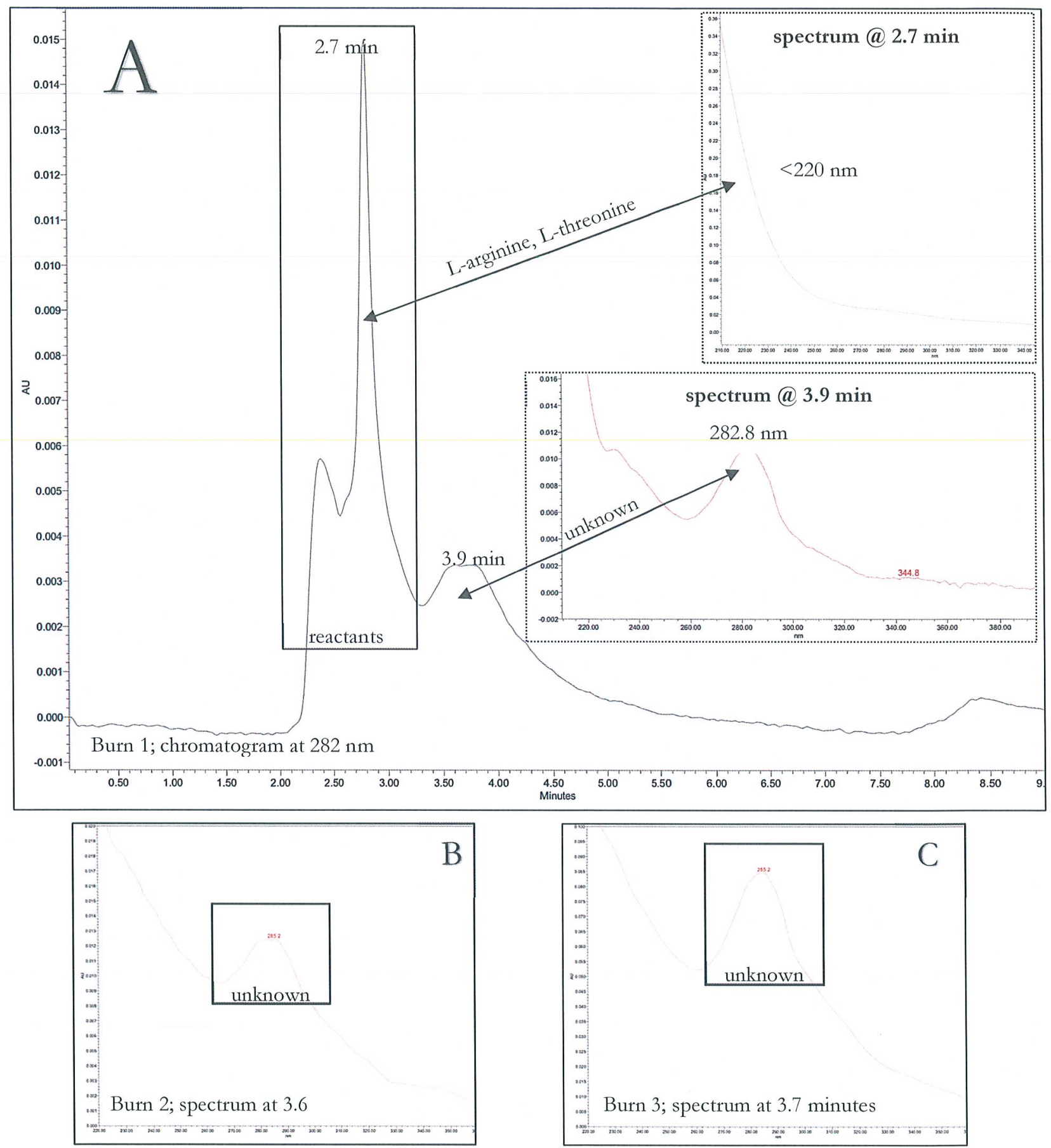

Figure 7: A) Chromatogram of burn 1 at $282 \mathrm{~nm}$; insets: burn 1 spectrum at 2.7 minutes, demonstrating the increasing absorbance below $220 \mathrm{~nm}$ due to unreacted amino acids; and burn 1 spectrum at 3.9 minutes, demonstrating the peak at $282.8 \mathrm{~nm}$ attributed to an unknown. B, C) Similarities in the UV-vis light absorption spectra of burns 2 and 3 at retention times 3.6 minutes and 3.7 minutes respectively, demonstrate the presence of unknown within these samples as well. 


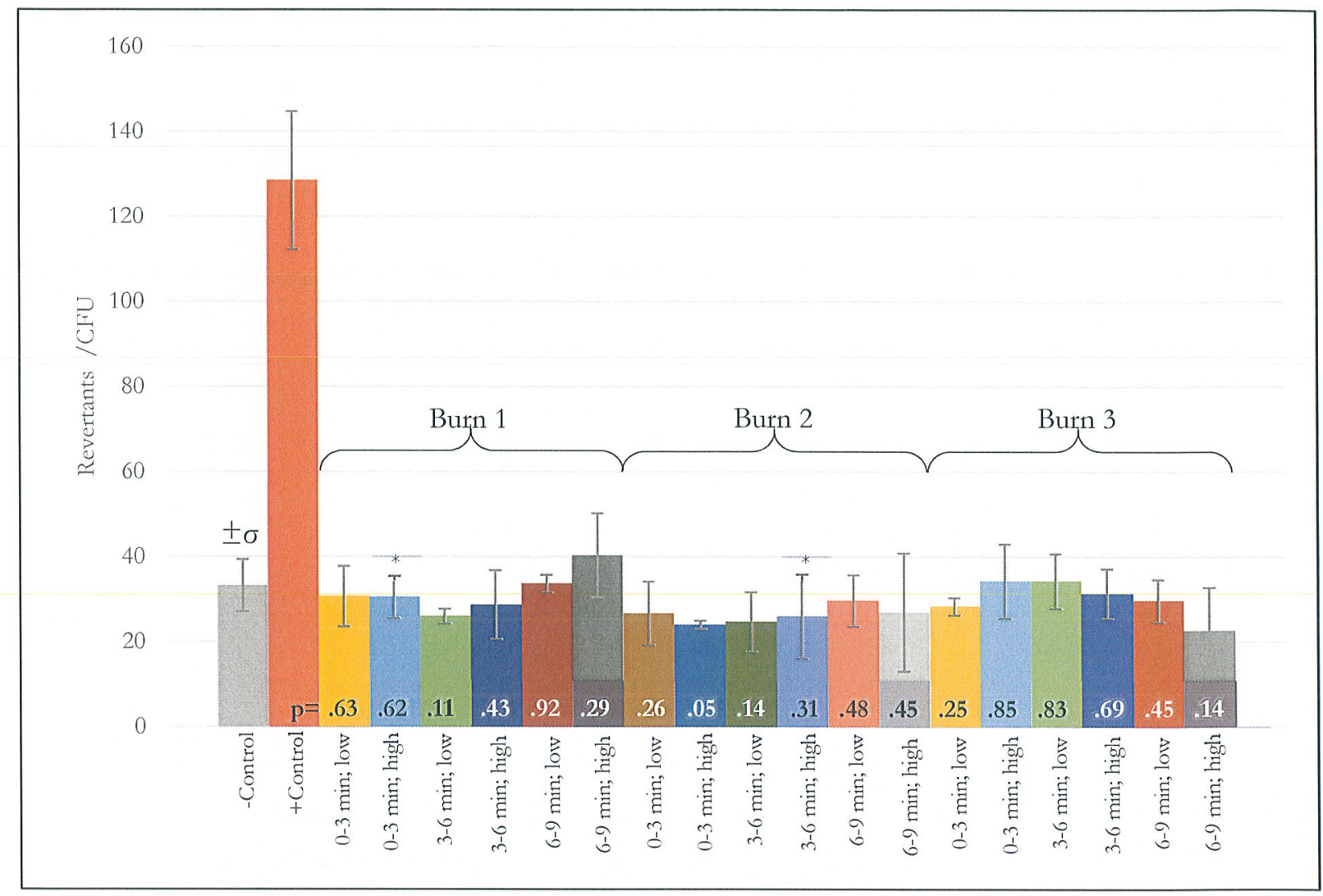

Figure 8: Ames mutagenicity test results for the 9 HPLC fractions. Results are grouped by burn, then by fraction. Results for both low and high dosages are shown. Asterisks denote results performed in duplicate instead of triplicate. p-values comparing the samples to the negative control are displayed at the base of each column

\section{Conclusion}

Burnings of creatine and L-phenylalanine resulted in the formation of $\mathrm{PhIP}$, validating our research methods. A positive control guaranteed that heterocyclic amines can be formed by our methodologies. This has been confirmed by several other researchers, using similar methods (Manabe et al, 1992; Pais et al, 1999). Furthermore, the amount of PhIP formed was enough to be detected by PrepHPLC, despite incredibly low burn yields. Thus, the methodology should be specific enough to determine the presence of a chemically similar novel heterocyclic amine from burnings of another binary amino acid mixture, but may not be sufficient to determine the molecular structure of the unknown. Future work should include modifications to the methodology to ensure a greater yield to prevent excessive waste of reagents.

PrepHPLC analysis of the burned binary amino acid mixture L-arginine and L-threonine demonstrated the presence of both unreacted amino acids arginine and threonine and an unknown compound eluting from the column at around 3.8 minutes, and absorbing maximally at $282 \mathrm{~nm}$; a UV-vis light absorption profile significantly different than PhIP. However, the compound is likely chemically similar to $\mathrm{PhIP}$, since both the unknown compound and $\mathrm{PhIP}$ were extracted from the burned samples using the same methodology, and both have similar retention times on the same column with the same mobile phase and flow rate. 
Although Knize et al. observe nearly 3200 revertants/mmol, a significant mutagenic activity, in the products of burnings of L-arginine and L-threonine when tested using the TA98 Salmonella Ames assay at doses between 0.2 and $0.4 \mathrm{mmol} /$ plate, our results disagreed with their observations (1994). A lack of significant difference between the samples tested and the negative solvent control, as well as a lack of dose response indicated that at the concentrations tested, neither the unreacted amino acids nor the unknown compound possessed mutagenic activity towards TA98 S9 activated Salmonella. However, that is not to say that the unknown compound is not at all mutagenic. The majority of HCA's are known to cause frameshift mutations (Alaejos \&Afonso, 2011). Despite this, Knize also reports a significant, but much smaller, mutagenic activity for the burned products of several binary amino acid mixtures using the TA100 strain instead. The TA100 Salmonella strain is a $\mathrm{G}: \mathrm{C} \rightarrow \mathrm{A}: \mathrm{T}$ sensitive strain (Knize et al, 1994; Koch et al, 1994). Performing the Ames test using a different strain of Salmonella may demonstrate that the unknown is indeed mutagenic, simply not towards the frameshift-sensitive TA98 strain. Increasing the dosage may also demonstrate the presence of mutagenicity; it is possible that the current doses are below a threshold for mutagenic activity.

No further analysis was performed on the extracts because no mutagenic activity was discovered. If future work leads to the discovery of mutagenic activity within the 3-6 minute fraction containing the unknown compound absorbing at $283 \mathrm{~nm}$, further steps towards isolation and purification should be taken. PrepHPLC or UltraHPLC analysis can be repeated using a modified mobile phase to achieve greater separation, and techniques such as IR and NMR may be used to elucidate a chemical structure (Chen et al, 2017; Hu et al, 2005).

\section{Acknowledgements}

Special thanks to Dr. Hayes, primary investigator and research supervisor for consistent guidance and helpful coaching, Dr. Brian Wong, professor at the Department of Biology, and his student researchers Kieun Chung, Snow Wan, and Joshua Li, for performing the Ames mutagenicity assay; Skyler Schell, who performed the amino acid burnings and initial extractions; and AU Office of Research and Creative Scholarship for providing financial support. 


\section{Works Cited}

Anand, P.; Kunnumakara, A.; Sundaram, C.; Harikumar, K.; Tharakan, S.; Lai, O.; Sung, B.; Aggarwal, B. Cancer is a Preventable Disease that Requires Major Lifestyle Changes. Pharm Res. 2008, 25(9), 2097-2116.

Alaejos, M.; Afonso, M. Factors that affect the content of heterocyclic aromatic amines in foods. Comprehensive Reviews in Food Science and Food Safety. 2011, 10, 52-108.

Chen, Jing; He, Z.; Qin, F.; Chen, Jie; Zeng, M. Formation of free and protein-bound heterocyclic amines in roast beef patties assessed by UPLC-MS/MS. Journal of Agricultural and Food Chemistry. 2017, 65, 4493-4499.

Felton, J.S.; Knize, M.G.; Shen, N.H.; Lewis, P.R.; Andresen, B.D.; Happe, J.; Hatch, F.T. The isolation and identification of a new mutagen from fried ground beef: 2-amino-1-methyl-6phenylimidazo[4,5-b]pyridine (PhIP). Carcinogenesis. 1986, 7(7), 1081-1086.

Gibis, M. Optimized HPLC Method for analysis of polar and nonpolar heterocyclic amines in cooked meat products. Journal of AO AC International. 2009, 92(3), 715-724.

Hu J.; Garo E.; Yoo H.; Cremin P.; Zeng L.; Goering M.; O’Neil-Johnson M.; Eldridge G. Application of Capillary-scale NMR for the Structure Determination of Phytochemicals. Phytochemical Analysis. 2005, 92, 127-133.

Knize, M.G.; Cunningham, P.L.; Avila, J.R.; Jones, A.L.; Griffin, Jr. E.A.; Felton, J.S. Characterization of mutagenic activity in cooked-grain-food products. Food and Chemical Toxicology. 1994, 32(1), 15-21.

Knize, M.G.; Cunningham, P.L.; Avila, J.R.; Jones, A.L.; Griffin, Jr. E.A.; Felton, J.S.; Formation of mutagenic activity from amino acids heated at cooking temperatures. Food and Chemical Toxicology. 1994, 32(1), 55-60.

Koch, W.; Henrikson, E.; Kupchella, E.; Cebula, T. Salmonella typhimurium strain TA100 differentiates several classes of carcinogens and mutagens by base substitution specificity. Carinogenesis. 1994, 15(1), 79-88.

Manabe, S.; Kurihara, N.; Wada, O.; Tohyama, K.; Aramaki, T. Formation of PhIP in a mixture of creatinine, phenylalanine and sugar or aldehyde by aqueous Heating. Carcinogenesis. 1992, 13(5), $827-$ 830 .

Pais, P.; Salmon, C.P.; Knize, M.G.; Felton, J.S. Formation of mutagenic/carcinogenic heterocyclic amines in dry-heated model systems, meats, and meat drippings. J. Agric. Food Chem. 1999, 47, 10981108.

Pender, T. Synthesis, isolation, and identification of carcinogenic heterocyclic amines using arginine. Andrews Univerisity. 2013. 
Puangsombat, K. Formation and Inhibition of Heterocyclic Amines in Meat Products. PhD Dissertation, Kansas State U, Manhattan, 2010.

Reichert, Z. M. Synthesis, isolation, and characterization of potentially carcinogenic arginine-based heterocyclic amines. Andrews University, 2016.

Schut, H.A.J; Snyderwine, E.G. DNA adducts of heterocyclic amine food mutagens: implications for mutagenesis and carcinogenesis. Carcinogenesis. 1999, 20, 353-368.

Sugimura T.; Wakabayashi K.; Nakagama K.; Nagao M. Heterocyclic amines: mutagens/carcinogens produced during cooking of meat and fish. Cancer Sci. 2004, 95, 290-99

Zheng, W.; Lee, S. Well-done meat intake, heterocyclic amine exposure, and cancer risk. Nutr. Cancer. 2009, 61, 437-446. 